\title{
The Effect of Open Lung Ventilation on Respiratory Mechanics, Postoperative Organ Function, and Inflammatory Mediators in Coronary Artery Bypass Surgery
}

\author{
Saloomeh Sehat Kashani' ${ }^{1}$, Faranak Rokhtabnak ${ }^{2}(\mathbb{D})$, Mohammad Reza Ghodraty ${ }^{2}(\mathbb{D})$, Mohammad \\ Mahdi Zamani $^{3,4,5}$ (D), Mehrdad Mesbah Kiaee ${ }^{1(D)}$, Masoud Ghorbanlo ${ }^{4,5}$, Shima movassaghi ${ }^{1}$, \\ Pooya Derakhshan ${ }^{1 *}[$ [D
}

1. Dept. of Anesthesiology, Rasoul Akram Hospital, Iran University of Medical Sciences, Tehran, Iran

2. Dept. of Anesthesiology, Firoozgar Hospital, Iran University of Medical Sciences, Tehran, Iran

3. Exceptional Talent Development Center (EDTC), Tehran University of Medical Sciences, Tehran, Iran

4. Dept. of Anesthesiology, School of Medicine, Iran University of Medical Sciences, Tehran, Iran

5. Hasheminejad Kidney Center (HKC), Iran University of Medical Sciences, Tehran, Iran

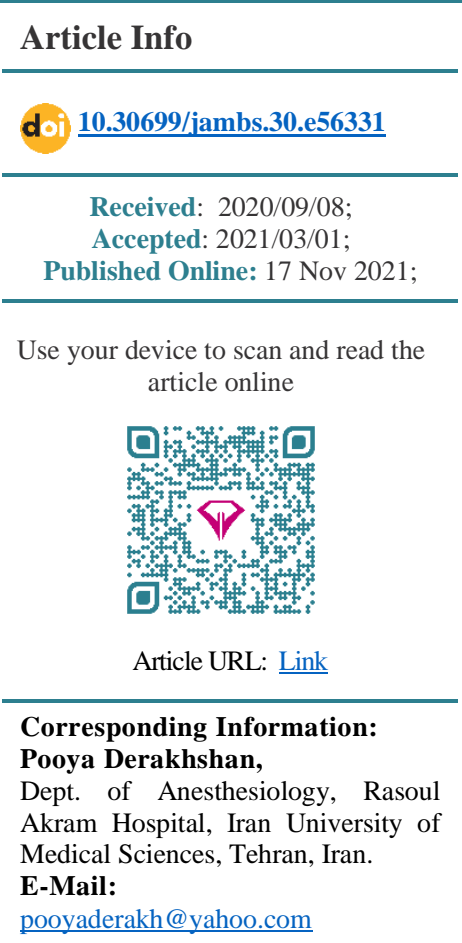

\begin{abstract}
Background \& Objective: The present study aimed to assess the supportive role of open lung ventilation on respiratory mechanics, the rate of oxygenation, inflammatory biomarkers, and probable liver or renal injuries following coronary artery bypass grafting surgery.

Materials \& Methods: This randomized double-blinded clinical trial study was conducted on 64 candidates for coronary artery bypass surgery using a cardiopulmonary pump. The patients were randomly categorized into the Positive endexpiratory pressure (PEEP) group $(\mathrm{n}=32)$ or Zero End Expiratory Pressure (ZEEP) group $(\mathrm{n}=32)$.

Results: Interleukin-6 levels were similar between the PEEP and ZEEP groups before surgery $(p=0.18)$ and were significantly higher in the ZEEP group after pump insertion $(p=0.005)$. On the contrary, the levels of Interleukin-6 were significantly higher in the PEEP group after extubation $(p=0.001)$. The Between-group analysis also showed a significant difference between the levels of interleukin-6 in the ZEEP and PEEP groups, representing a greater increase in the PEEP group $(p<0.001)$. There was no difference in certain hemodynamic parameters, including heart rate, mean blood pressure, mean $\mathrm{CO} 2$ pressure (PCO2), mean concentration of $\mathrm{HCO} 3$, and base excess. The mean arterial $\mathrm{O} 2$ saturation was higher overall in the PEEP group compared to the ZEEP group. The mean $\mathrm{PaO} 2 / \mathrm{FiO} 2$ was significantly higher in the PEEP than in the ZEEP group $(\mathrm{p}<0.001)$.
\end{abstract}

Conclusion: Supportive ventilation technique leads to better oxygenation and better lung expansion, as well as lowering inflammatory biomarkers, after coronary artery bypass surgery.

Keywords: Coronary Artery Bypass, Pulmonary Ventilation, Interleukin-6, Positive-Pressure Respiration

\section{Introduction}

Respiratory failure after major surgeries, especially cardiopulmonary bypass operations, is an important clinical issue, leading to subclinical functional changes in most affected patients and even acute respiratory distress syndrome in about two percent of patients who undergo coronary artery bypass grafting (CABG) surgery $(\mathbf{1 , 2})$. Common physiological disturbances following this surgery are manifested by abnormal changes in gas exchange, as well as abnormal mechanical changes in the lung, leading to reduced tissue oxygenation and even acute respiratory failure (3). In these conditions, utilizing mechanical ventilation could increase the positive pressure in the chest and subsequently reduces cardiac preload, leading to decreases in cardiac output and blood pressure; which could induce reactive shock to fluid therapy (3). In addition, the increase in intra-thoracic pressure can also reduce the glomerular filtration rate (GFR) (4). Moreover, applying mechanical ventilation may change neuro-hormonal pathways, such as the reninangiotensin pathway, leading to released vasopressin and atrial natriuretic peptide (5). In this regard, it is now suggested that pro-inflammatory cytokines such as 
interleukin 1,6 , and 8 , as well as $\mathrm{TNF} \alpha$, may play central roles in acute kidney injury (6).

Recently, open lung ventilation which has been defined as a low tidal volume plus relatively high positive endexpiratory pressure has been accepted as a supportive strategy to prevent lung atelectasis (due to shear forces during major surgeries, which increase the risk of atelectatic lung events) (7). This strategy can increase intrathoracic pressure, but it increases the right ventricular afterload due to alveolar over-distention of the lung, in the presence of atelectasis or hypoxic vasoconstriction, which leads to reduced surgical safety (8). It has been recently hypothesized that by using open lung ventilation, the increase in the right ventricular afterload can be avoided in cardiac surgeries (9). Furthermore, employing this approach may result in alleviated respiratory-related complications in this surgery, probably due to reduced inflammatory biomarkers (10). This study aimed to assess the supportive role of open lung ventilation on respiratory mechanics, the rate of oxygenation, inflammatory biomarkers, and probable liver or renal injuries following CABG surgery.

\section{Materials and Methods}

This randomized double-blinded clinical trial was performed on 64 candidates for CABG surgery using a cardiopulmonary pump who had been referred to Hasheminejad Hospital, Tehran between January 2013 and January 2014. All eligible patients were aged 18 to 70 years, candidates for surgery under general anesthesia, with a body mass index lower than $30 \mathrm{~kg} / \mathrm{m}^{2}$, without a history of obesity, and agreed with performing respiratory functional tests. The exclusion criteria were defined as candidates for emergency surgery, a history of lung surgeries, a history of chronic obstructive lung disease, repeated treatment with systemic corticosteroids, a history of liver or kidney diseases, drug or alcohol abuse, sensitivity to anesthetic drugs, receiving radiotherapy or immunosuppressive drugs (including chemotherapy agents) within the last two months, serum hemoglobin level of less than $10 \mathrm{mg} / \mathrm{dl}$ or albumin level of less than 3 $\mathrm{gr} / \mathrm{dl}$, hemodynamic instability or persistent shock, or a predicted need for prolonged mechanical ventilation (longer than 6 hours after surgery). The present trial was approved by the ethical committee at Iran University of Medical Sciences (code 1394.9111174018) and written consent was obtained from all patients, which was registered at Iran Registry of Clinical Trials (IRCT code: IRCT2015040115774N3).

The patients were randomly categorized (using the block randomization method) into the PEEP group ( $\mathrm{n}=$ $32)$ or the ZEEP group $(n=32)$. On entrance to the operating room, the patients, were monitored by the standard protocol (electrocardiogram, pulse oximetry, IBP, and ETCO2). All subjects were pre-medicated with lorazepam (1mg, IV) two hours before surgery and morphine sulfate $(0.1$ to $0.5 \mathrm{mg} / \mathrm{kg}$, IM) 30 minutes before transfer to the room. All patients were generally anesthetized using etomidate $(0.15$ to $0.30 \mathrm{mg} / \mathrm{kg})$ plus fentanyl (3 to $10 \mathrm{mcg} / \mathrm{kg}$ ), lidocaine (1.0 to $1.5 \mathrm{mg} / \mathrm{dl}$ ), then atracurium $(0.15$ to $0.20 \mathrm{mg} / \mathrm{kg})$. Esophageal tube cuff pressure was maintained at 20 to $25 \mathrm{mmHg}$. Anesthesia was maintained using midazolam ( 0.25 to 0.5 $\mathrm{mcg} / \mathrm{kg} / \mathrm{min})$ and fentanyl (0.03 to $0.1 \mathrm{mcg} / \mathrm{kg} / \mathrm{min})$ and then atracurium $(0.03 \mathrm{mg} / \mathrm{kg})$. All hemodynamic parameters were monitored before anesthesia and during operation every 15 minutes, on Intensive Care Unit (ICU) admission, and also during extubation (including heart rate, mean blood pressure, mean arterial oxygen saturation, expiratory $\mathrm{CO}_{2}$ pressure, respiratory rate, tidal volume, arterial blood gas, static and dynamic compliance). Moreover, urine output, the volume of bleeding during the operation, total time of operation, volume of infused fluid, time for balloon pump insertion, and time for intubation were also measured. In the PEEP group, positive end-expiratory pressure of $10 \mathrm{cmH}_{2} \mathrm{O}$ and tidal volume of $6 \mathrm{cc} / \mathrm{kg}$ were considered, and respiratory rate was set on $\mathrm{EtCO}_{2}$ ranging from 30 to $35 \mathrm{mmHg}$, with similar orders continued at the ICU ward. In the ZEEP group, positive end-expiratory pressure was maintained at 0 , tidal volume at $9 \mathrm{cc} / \mathrm{kg}$, and respiratory rate on $\mathrm{EtCO}_{2}$ ranging from 30 to $35 \mathrm{mmHg}$, with similar orders continued at the ICU. 24 hours after surgery, all liver and renal function parameters, coagulation factors, fasting blood sugar, and interleukin-6 levels were reassessed.

For statistical analysis, results were presented as mean \pm standard deviation (SD) for quantitative variables and were shorted by absolute frequencies and percentages for categorical variables. The normality of data was analyzed by the Kolmogorov-Smirnoff test. Categorical variables were compared by chi-square test, or Fisher's exact test after more than $20 \%$ of cells with an expected amount of less than 5 were observed. Quantitative variables compared with T-test or Mann-Whitney U-test. The tendency of the change in the levels of interleukin- 6 at different time points was measured using the Repeated Measure ANOVA test and the Greenhouse-Geisser correlation test. For statistical analysis, SPSS version 16.0 for Windows (SPSS Inc., Chicago, IL) was used. $p$-values of 0.05 or less were considered statistically significant.

\section{Results}

In total, one patient in the PEEP group was excluded from the study due to later extubation as a result of high chest tube drainage. Similarly, two patients in the ZEEP group were also excluded because of tachypnea and metabolic acidosis leading to extubation beyond 6 hours. As shown in Table 1, the two groups were similar in gender, mean age, mean body mass index, mean operation time, the mean time for using a balloon pump, the volume of bleeding, urine output, and time for extubation. The levels of interleukin- 6 were similar between the two groups before surgery $(p=0.18)$, then significantly higher in the ZEEP group after pump insertion $(p=0.005)$, and were conversely higher in the PEEP group after extubation $(p=0.001)$ (Table 2). The Greenhouse-Geisser test showed a significant correlation between the type of ventilation and the levels of interleukin- $6(p<0.001)$. the 
Between-group analysis also showed a significant difference in the levels of interleukin- 6 between the ZEEP and PEEP groups representing a greater increase in the PEEP group ( $\mathrm{p}<0.001$ ) (Figure 1). There was no difference in certain hemodynamic parameters, including heart rate, the mean blood pressure, mean $\mathrm{PCO}_{2}$ pressure, mean concentration of $\mathrm{HCO}_{3}$, and base excess. However, the Greenhouse-Geisser analysis showed the significant effect of the type of ventilation on arterial $\mathrm{O}_{2}$ saturation within each group $(\mathrm{p}<0.001)$, as well as a significant between-group difference in this parameter $(\mathrm{p}<0.001)$. In other words, mean arterial $\mathrm{O}_{2}$ saturation was overall higher in the PEEP group as compared to the ZEEP group (Figure 2). Regarding the difference in compliance, higher static compliance was revealed in the PEEP group than in the ZEEP group $(\mathrm{p}=0.005)$, however, no difference was found in dynamic compliance between the groups $(\mathrm{p}=$ 0.059). The mean $\mathrm{PaO}_{2} / \mathrm{FiO}_{2}$ was significantly higher in the PEEP group in comparison with the ZEEP group $(\mathrm{p}<$ $0.001)$.

Table 1. Comparison of baseline variables between the ZEEP and PEEP groups

\begin{tabular}{|lccc|}
\hline Variable & PEEP group & ZEEP group & p-value \\
\hline Female gender & $17(53.1)$ & $16(50.0)$ & 0.89 \\
\hline Mean age, year & $56.33 \pm 9.28$ & $56.72 \pm 8.11$ & 0.92 \\
\hline Mean BMI, kg/m2 & $26.00 \pm 1.51$ & $25.47 \pm 1.39$ & 0.66 \\
\hline Duration of surgery, min & $167.65 \pm 24.95$ & $165.31 \pm 19.79$ & 0.67 \\
\hline Time on balloon pump, min & $39.50 \pm 10.68$ & $37.34 \pm 10.31$ & 0.41 \\
\hline Volume of bleeding, ml & $384.37 \pm 121.44$ & $404.68 \pm 137.58$ & 0.53 \\
\hline Urine output, ml & $1092.18 \pm 343.39$ & $1020.31 \pm 290.40$ & 0.36 \\
\hline Intubation time, min & $305.00 \pm 43.83$ & $293.84 \pm 42.05$ & 0.36 \\
\hline
\end{tabular}

Table 2: Comparison ofinterleukin-6 levels between ZEEP and PEEP groups

\begin{tabular}{lccc} 
Variable & PEEP group & ZEEP group & \multicolumn{1}{c}{ p-value } \\
\hline Before surgery & $6.67 \pm 2.49$ & $5.33 \pm 2.23$ & 0.18 \\
\hline After balloon pumping & $34.75 \pm 14.64$ & $59.50 \pm 22.83$ & 0.005 \\
\hline After extubation & $87.75 \pm 22.57$ & $57.17 \pm 14.97$ & 0.001 \\
\hline
\end{tabular}

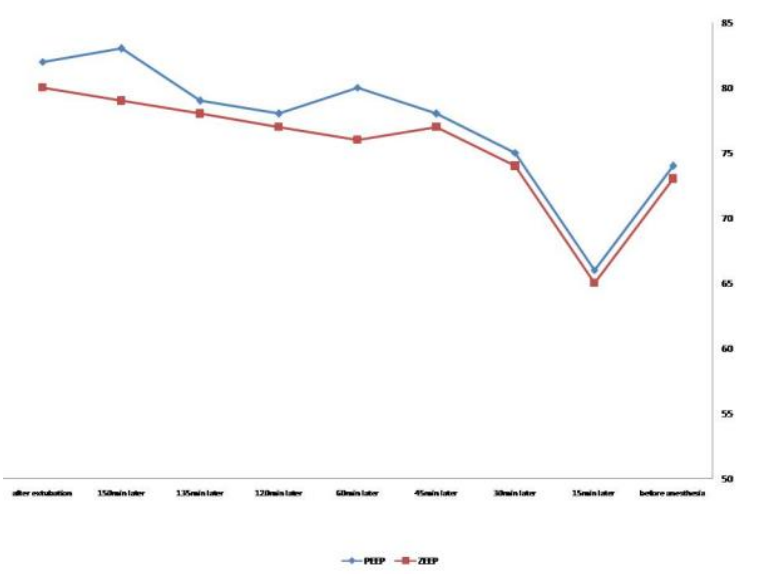

Figure 1. Trend of the changes in interleukin-6 levels in the PEEP and ZEEP groups

\section{Discussion}

The present study shows that the application of low tidal volume along with end-expiratory pressure, within

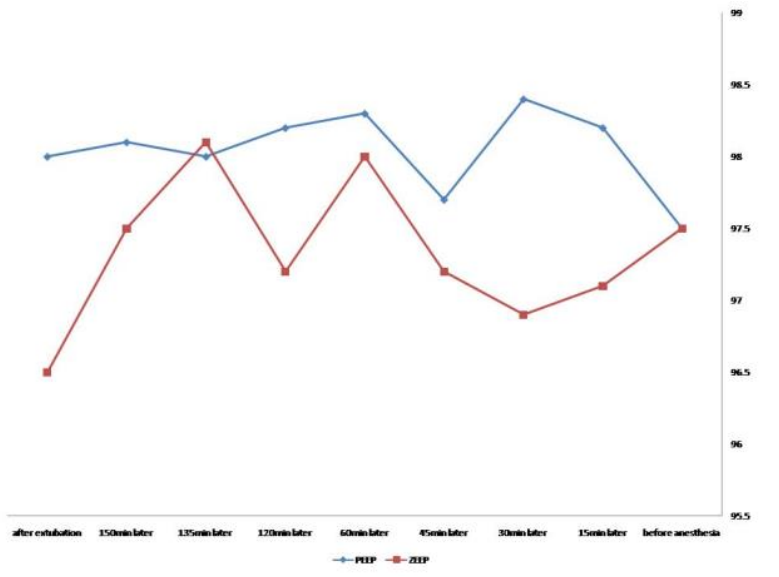

Figure 2. Trend of the changes in arterial $\mathrm{O2}$ saturation in the PEEP and ZEEP groups

coronary artery bypass surgery as a supportive protocol, leads to better oxygenation, improves lung expansion, 
besides lowering inflammatory biomarkers. According to recent reports, employing mechanical ventilation with high tidal volume may activate inflammatory pathways within major surgeries. The current study clearly indicates that using conventional ventilation could increase the serum levels of interleukin-6, indicating an increased risk for lung injuries, due to repeatedly collapsing the lungs during mechanical ventilation. Similar to our findings, Patel et al. showed an increase in the levels of interleukin6 after both ventilation approaches, including conventional and supportive methods; however, this increase was lower in the supportive approach (11). In another survey by von Bethmann et al., interleukin-6 was introduced as a specific marker for assessing the progression of lung injuries during major surgeries (12). Similarly, Zupancich et al. and Lin et al. revealed that the level of this marker was considerably lower in those who received the supportive ventilation method $(13,14)$. It seems that by inhibiting the inflammatory pathways mediated by the decrease in inflammatory biomarkers such as interleukin-6, lung injuries mediated by inflammation can be significantly reduced. Independent of inflammatory mechanisms, mechanical ventilation can induces lung injuries via increasing intra-thoracic pressure, as well as decreasing the glomerular filtration rate (GFR). Based on the present results, the conventional ventilation approach induce augmentation in serum creatinine levels. As shown by Imai et al., the increase in inflammatory cytokines can cause cell apoptosis in the kidneys (15). In other words, interleukin-6 can concurrently predict both injuries in lungs and kidneys following ventilation, which is less expected in the conventional ventilation approach.

The present study also showed no difference in mean arterial blood pressure between the two conventional and supportive approaches, despite the study by Zupancich et al. (13) However, the present data found higher arterial oxygenation and also higher $\mathrm{PiO}_{2} / \mathrm{FiO}_{2}$ following the supportive approach, indicating better oxygenation in those scheduling lower tidal volume. DYHR et al. pointed out a direct association between better oxygenation and using supportive ventilation (16). In fact, applying PEEP at the end of the expiratory period leads to higher static compliance. However, this benefit was specified to only static but not dynamic compliance, similar to Chaney et al.'s study, (17) but contrary to Lin et al.'s study (14).

\section{Conclusion:}

In total, it can be seen that the use of low tidal volume, along with end-expiratory pressure, within coronary artery bypass graft surgery as a supportive protocol could ameliorate oxygenation, lung expansion, together with a reduction in inflammatory biomarkers.

\section{Acknowledgment:}

This study has been granted by Iran University of Medical Sciences.

\section{Conflicts of Interest:}

There is no conflict of interest.

\section{References}

1. Canver CC, Chanda J. Intraoperative and postoperative risk factors for respiratory failure after coronary bypass. Ann ThoracSurg. 2003; 75(3):853-7. 4975(02)04493-4]

2. Faritous ZS, Aghdaie N, Yazdanian F, Azarfarin R, Dabbagh A. Perioperative risk factors for prolonged mechanical ventilation and tracheostomy in women undergoing coronary artery bypass graft with cardiopulmonary bypass. Saudi J Anaesth. 2011; 5:167-9. [DOI:10.4103/1658-354X.82786] [PMID] [PMCID]

3. Cournand A, Motley HL, Werko L, Richards D. Physiological studies of the effects of intermittent positive pressure breathing on cardiac output in man. Am J Physiol. 1948; 152(1):162-174. [DOI:10.1152/ajplegacy.1947.152.1.162] [PMID]

4. Koyner JL, Murray PT. Mechanical ventilation and the kidney. Blood Purif. 2010; 29(1):52-68. [DOI:10.1159/000259585] [PMID] [PMCID]

5. Annat MDG, Viale MDJP, Xuan BB, Aissa MDOH, Benzoni PDD, Vincent PDM, et al. Effect of PEEP Ventilation on Renal Function, Plasma Renin, Aldosterone, Neurophysins and Urinary ADH, and Prostaglandins. Anesthesiology. 1983; 58(2):136-41. [DOI:10.1097/00000542198302000-00006] [PMID]

6. Ferrante A, Nandoskar M, Walz A, Goh DH, Kowanko IC. Effects of tumour necrosis factor alpha and interleukin-1 alpha and beta on human neutrophil migration, respiratory burst and degranulation. Int Arch Allergy Immunol. 1988; 86(1):82-91. [DOI:10.1159/000234610] [PMID]

7. Slinger P, Kilpatrick B. Perioperative Lung Protection Strategies in Cardiothoracic Anesthesia. Anesthesiol Clin. 2012; 30(4):607-28. [DOI:10.1016/j.anclin.2012.07.001] [PMID]

8. Habashi NM. Other approaches to open-lung ventilation: airway pressure release ventilation. Crit Care Med. 2005; 33(3):S228-40. [DOI:10.1097/01.CCM.0000155920.11893.37] [PMID]

9. Biondi JW, Schulman DS, Soufer R, Matthay RA, Hines RL, Kay HR, etal. The effect of incremental positive end-expiratory pressure on right ventricular hemodynamics and ejection fraction. AnesthAnalg. 1988; 67(2):144-151. [DOI:10.1213/00000539-198802000-00007]

10. Maggiore SM, Jonson B, Richard JC, Jaber S, Lemaire F, Brochard L. Alveolar derecruitment at 
decremental positive end-expiratory pressure levels in acute lung injury: comparison with the lower inflection point, oxygenation, and compliance. Am J RespirCrit Care Med. 2001; 164(5):795-801.

\section{[DOI:10.1164/ajrccm.164.5.2006071] [PMID]}

11. Patel JM. Intra-operative adherence to lungprotective ventilation: a prospective observational study. Perioper Med (Lond). 2016; 5: 8. [DOI:10.1186/s13741-016-0033-4] [PMID] [PMCID]

12. von Bethmann AN, Brasch F, Nusing R, Vogt K, Volk HD, Muller KM, et al. Hyperventilation induces release of cytokines from perfused mouse lung. Am J Respir Crit Care Med. 1998; 157(1):263-72.

[DOI:10.1164/ajrccm.157.1.9608052] [PMID]

13. Zupancich E, Paparella D, Turani F, Munch C, Rossi A, Massaccesi S, et al. Mechanical ventilation affects inflammatory mediators in patients undergoing cardiopulmonary bypass for cardiac surgery: a randomized clinical trial. J Thorac Cardiovasc Surg. 2005;130(2):378-83. [DOI:10.1016/j.jtcvs.2004.11.061] [PMID]
14. Lin WQ, Lu XY, Cao LH, Wen LL, Bai XH, Zhong ZJ. [Effects of the lung protective ventilatory strategy on proinflammatory cytokine release during one-lung ventilation]. Ai Zheng. 2008;27(8):870-3.

15. Imai $Y$, Kawano $T$, Iwamoto $S$, Nakagawa $S$, Takata M, Miyasaka K. Intratracheal anti-tumor necrosis factor-alpha antibody attenuates ventilator-induced lung injury in rabbits. J Appl Physiol. 1999;87(2):510-5. [DOI:10.1152/jappl.1999.87.2.510] [PMID]

16. Dyhr T, Laursen N, Larsson A. Effects of lung recruitment maneuver and positive end-expiratory pressure on lung volume, respiratory mechanics and alveolar gas mixing in patients ventilated after cardiac surgery. Acta Anaesthesiol Scand. 2002;46(6):717-25. [DOI:10.1034/j.13996576.2002.460615.x] [PMID]

17. Chaney MA, Nikolov MP, Blakeman BP, Bakhos M. Protective ventilation attenuates postoperative pulmonary dysfunction in patients undergoing cardiopulmonary bypass. J Cardiothorac Vasc Anesth. 2000;14(5):514-8

[DOI:10.1053/jcan.2000.9487] [PMID]

\section{How to Cite This Article:}

\section{Sehat Kashani S, Rokhtabnak F, Ghodraty M R, Zamani M M, Mesbah Kiaee M, Ghorbanlo M, et al . The Effect of Open Lung Ventilation on Respiratory Mechanics, Postoperative Organ Function, and Inflammatory Mediators in Coronary Artery Bypass Surgery. J Adv Med Biomed Res. 2022; 30 (138): 12-16}

\section{Download citation:}

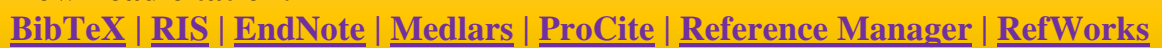

